\title{
SCEA: A Speed-Changing based Algorithm to Optimize Decision Time for Emergency Rescue
}

\author{
Qinyong $\mathrm{Li}^{1+}$, Haoming Guo ${ }^{1}$, Xinjian Shan ${ }^{2}$, Yanyan $\mathrm{Wei}^{1}$, Zhixin Wang ${ }^{1}$, Jianxiu Bai ${ }^{1}$ and \\ Qiuhong Zhang ${ }^{1}$ \\ ${ }^{1}$ Center of Collaborative Innovation, Institute of Software, China Academy of Science, China \\ ${ }^{2}$ State Key Laboratory of Earthquake Dynamics, Institute of Geology, China Earthquake Administration, \\ China
}

\begin{abstract}
Decision time optimizing is a core problem during the process of disaster emergency response. Traditional methods are mainly based on expert experiences, which is lack objectiveness and error prone. In this paper, based on the practical disaster assessment results and the disaster model we built, we propose two algorithms, DCEA and SCEA, to cope with the problem. DCEA is a distance-based algorithm to convert disaster assessment results series to unevenly time series. In this algorithm, we utilize Hausdorff distance as the distance operator. Compared to DCEA, an optimized speed-changing based algorithm SCEA is proposed. Experiments on real earthquake disaster events demonstrate the effectiveness and superiority of proposed work.
\end{abstract}

Keywords: Decision Support System, Time Series Analysis, Disaster Emergency Response

\section{Introduction}

Natural disasters cause enormous loss to people's production and livelihoods. Efficient emergency decision-making is an important tool for mitigating the impact of disasters. Effective decision making is based on an effective assessment of the disaster impact [1]. According to the general rules for obtaining data during disaster, during the Black Box period, the disaster assessment results are not objective due to the lack of reliable data. With the availability of high-quality data, the results of disaster assessments are becoming more objective. However, due to the strong time-constraints of disaster emergency decisions, the effectiveness of rescue will be greatly reduced if the decision time misses the best time to rescue. [2]At present, the determination of the time point for emergency decision making is generally done manually or by the experience of experts, which relies entirely on the experience rather than the situation of the disaster, resulting errors in decision making[3].

In this paper, we address the problem of optimizing time-constraints for disaster emergency decision making in a big data environment by proposing the concept of the convergence of Disaster Assessment Result Series (DARS) [4][5] and related algorithms. We firstly give the definition of Disaster Event Model and the definition convergence of DARS. Based on these definitions, we further indicate the conditions for determining the convergence of DARS. We propose two algorithms, which are DCEA and SCEA, to extract the convergence trends in DARS. Finally, we validate the effectiveness of proposed algorithms using a group of real earthquake events and associated DARS.

\section{Definitions}

\subsection{Disaster Event Model}

The Disaster Event can be denoted as a five-tuple structure as follows:

$$
D E=(\text { time }, \text { loc, obj, class, content })
$$

\footnotetext{
+ Corresponding author. Tel.: + 86-010-62661192; fax: +86-010-62661192.

E-mail address: qinyong @iscas.ac.cn
} 
Among the definition, time indicates the moment of the disaster occur; loc represents the location of the disaster, obj represents the related objects during the process during the disaster response, and class represents the disaster information classification, such as death number, disaster region .etc; content $=\left\{c_{0}, c_{1}, \ldots, c_{N_{A}}\right\}$ represents the content related disaster information. Disaster information includes not only the basic semantics but also the temporal semantics.

We define $D E O$ as the initial state of the disaster event. It is not depending on the disaster information. In the process of disaster response, it can be user-generated or monitored in real time through an automatic alarm mechanism.

\subsection{Disaster Assessment Result Series}

Given a series of time point $T=<t_{0}, t_{1}, \ldots, t_{k}, \ldots, t_{N}>$ which represent the moment passed after disaster $D E$ occurred. $t_{0}$ is the occur moment of $\mathrm{DE}$ and $\mathrm{N}$ is a natural number that are large enough. $M^{t_{i}}$ is the group of disaster analysis models and $D^{t_{i}}$ represents the data source set at the moment $t_{i}$.

The process of disaster estimation PDE can be denoted as a monotone bounded function $S\left(M^{t_{i}}, D^{t_{i}}, T\right)$. At any moment in $t \in T, i=1,2, \ldots, N$, disaster estimation system uses a range of analysis models and data sources to assess the impact of disaster and in turn get the disaster assessment results. Namely, $S\left(M^{t_{i}}, D^{t_{i}}, T\right)=R^{t_{i}}$. As a result, we can obtain a series of DAR, which denoted as $R=<R_{0}, R_{1}, \ldots, R_{N}>$.

\subsection{Convergence of DARS}

Given a disaster event DE and related DARS at moments $T=<t_{0}, t_{1}, \ldots, t_{i}, \ldots, t_{i+1} \ldots, t_{N}>$, which denoted as $R=<R_{0}, R_{1}, \ldots, R_{n}>$. The upper-limit and lower limit of $R$ are represented as in equation (2) and equation (3):

$$
\begin{aligned}
& \overline{\lim _{i \rightarrow \infty} R_{t_{i}}}=\bigcap_{i=1}^{\infty} \cup_{j=i}^{\infty} R_{j} \\
& \underline{\underline{\lim _{i \rightarrow \infty} R_{i}}}=\cup_{i=1}^{\infty} \bigcap_{j=i}^{\infty} R_{j}
\end{aligned}
$$

We define that the DARS $R=<R_{0}, R_{1}, \ldots, R_{n}>$ is convergent iff. both upper and lower limit existed and $\overline{\lim _{i \rightarrow \infty} R_{t_{i}}}=\lim _{i \rightarrow \infty} R_{i}=\Phi$. Further, the convergent condition of DARS is proposed as follows:

$$
\lim _{t \rightarrow \infty} \operatorname{DIST}\left(\left|R_{T_{j}}-R_{T_{i}}\right|\right) \leq \epsilon, j>i
$$

In equation (4), $T_{j}$ and $T_{i}$ represent different time periods, $R_{T_{j}}$ and $R_{T_{i}}$ are assessment results at $T_{j}, T_{i}$. $\operatorname{DIST}(\cdot)$ is a distance operator to measure the similarity between $R_{T_{j}}$ and $R_{T_{i}}, \epsilon$ is the threshold factor. We can draw a conclusion that the DARS is convergent when $t \rightarrow \infty$ and the similarity between $R_{T_{j}}$ and $R_{T_{i}}$ is less than $\epsilon$.

\section{Algorithms}

In this section, we describe two algorithms, which are DCEA and SCEA, to extract convergence trend in DARS. SCEA is an enhanced version of DCEA.

\subsection{DCEA: Distance-based Convergence Extraction Algorithm for DARS}

To depict the time-constraints of disaster response, the basic idea of DCEA is that using some sort of distance between DAR to extract the convergence trend of DARS. Suppose that $S_{i}, S_{i+1}$ are the value set of $R_{t_{i}}, R_{t_{i}+1}$, respectively. The process to calculate the distance between $S_{i}$ and $S_{i+1}$ is mainly depends on the operator of distance. The DCEA algorithm is given in Algorithm I. 


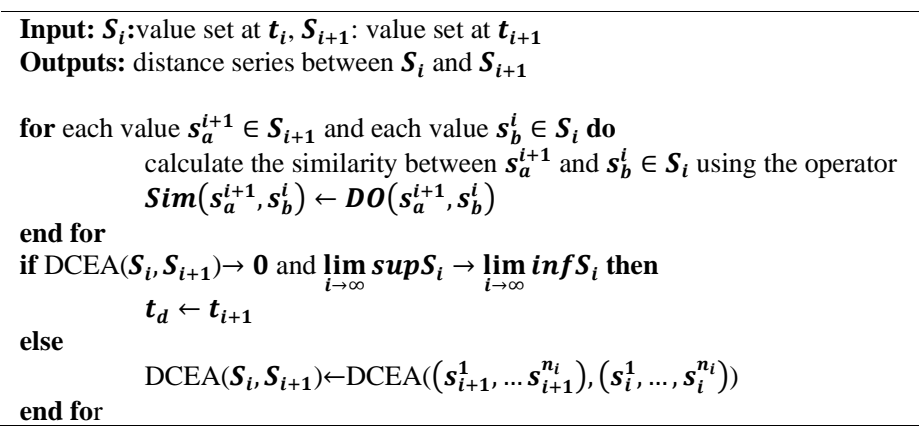

DCEA is a recursion algorithm according to the process described above. The algorithm considers the moment when DARS is convergent is the optimal timestamp to make decision.

The un-decided part in DCEA is the distance operator. The main effect of distance operator is to compute the similarity between DAR, which could be structured data, semi-structured data and even unstructured data. In this paper, we utilize Hausdorff Distance as the distance operator of DCEA.

Hausdorff distance describes a difference measure between two finite closed sets without requiring that the two closures have the same number of elements. This characteristic is very suitable for calculating the similarity between DAR. The Hausdorff distance is calculated as shown in equation (5):

$$
H(A, B)=\max (h(A, B), h(B, A))
$$

In equation (5), $H(A, B)$ is called the two-way Hausdorff distance between set $A$ and $B . h(A, B)$ and $h(B, A)$ is called one-way Hausdorff distance.

$$
\begin{aligned}
& h(A, B)=\max _{a \in A} \min _{b \in B}|| a-b|| \\
& h(A, B)=\max _{b \in B} \min _{a \in A}|| a-b||
\end{aligned}
$$

where operator $\|\cdot\|$ is distance $p$-modulus:

$$
d_{12}=\sqrt[p]{\sum_{k=1}^{n}\left|x_{1 k}-x_{2 k}\right|^{p}}
$$

The DCEA algorithm uses Hausdorff distance as the operator to convert DARS to a time series. So, the development and the evolution process of disaster events can be visualized as a curve. However, there are still some practical problems:

1) How to remove the noise component from the series?

2) The DCEA captures the trend between adjacent moments in the sequence and does less to depict the overall trend over a time horizon.

3) The DCEA traces the convergence of sequence of DARS by distance operator, the obtained result cannot adequately describe the objective trend of the sequence of DARS.

To cope with above problems, based on the slide window methods in time series analysis, we propose the speed-changing based algorithm, which is the speed-changing based convergence extraction algorithm (SCEA).

\subsection{SCEA: Speed-changing based Convergence Extraction Algorithm for DARS}

Given a disaster event $D E$, and the timestamp series $t_{0}, t_{1}, \ldots, t_{i}, t_{i+1}, \ldots, t_{N}$, which $t_{0}$ represents the moment disaster occurred on. $R_{0}, R_{1}, \ldots, R_{i}, R_{i+1}, \ldots$ represents related disaster assessment results. We convert DARS $R_{0}, R_{1}, \ldots, R_{i}, R_{i+1}, \ldots$ to a value-based time series $X=\left\{x_{1}, x_{2}, \ldots, x_{n}\right\}$, where $x_{i}=$ $\operatorname{DCEA}\left(R_{i}, R_{i+1}\right), i=1,2, \ldots, n . X=\left\{x_{1}, x_{2}, \ldots, x_{n}\right\}$ is an unevenly time series. 


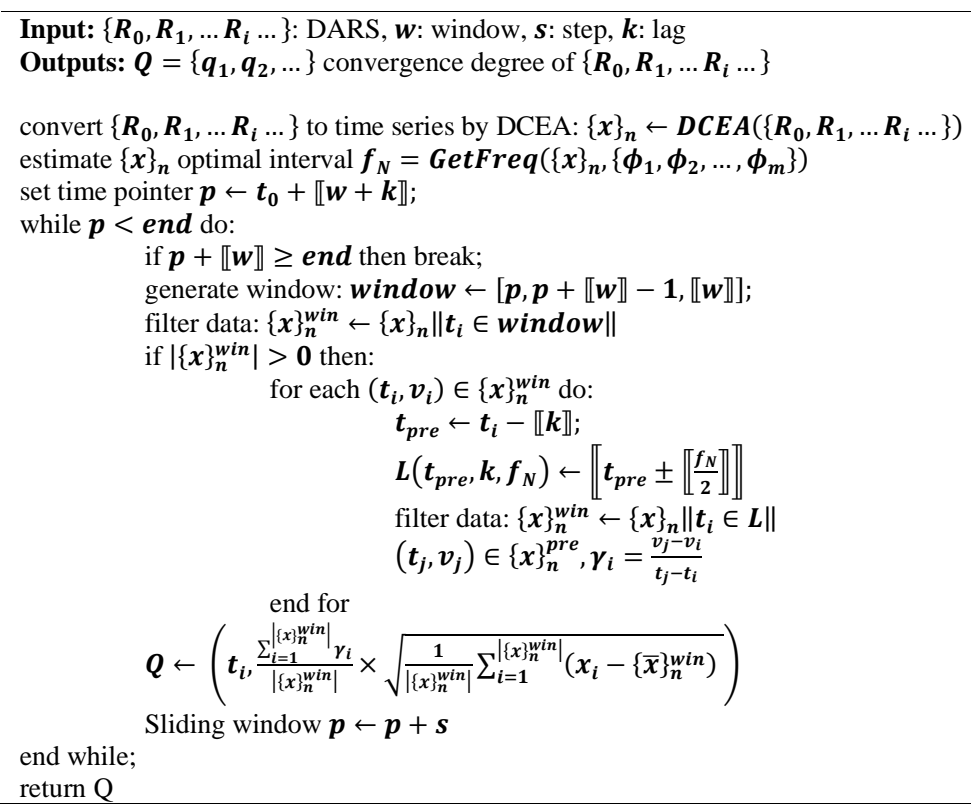

Given a time window $w$ with length $|w|$, the sub series in $X$ which captured by $w$ can be denoted as $Y=$ $\left\{y_{1}, y_{2}, \ldots, y_{w}\right\}, Y \subseteq X$. The convergence degree of $Y$ can be denoted as:

$$
q(Y)=\sigma_{Y} * \overline{\gamma_{Y}}
$$

The convergence degree of DARS is described as two parts: $\sigma_{Y}$ and $\overline{\gamma_{Y}} \cdot \sigma_{Y}$ is the standard deviation in time window $w \cdot \bar{\gamma}_{Y}$ is the average speed value in time window $w$.Arguments $k$ and $f$ are the lag and the optimal interval. The SCEA is proposed in Algorithm II.

Suppose that the disaster occurred timestamp is $t_{0}$ and current timestamp is $t_{n+1}$. Disaster assessment series can be obtained as $\left\{R_{1}, R_{2}, \ldots, R_{n+1}\right\}$. The inputs of SCEA are $\left\{R_{1}, R_{2}, \ldots, R_{n+1}\right\}$, time window $w$ and step $S$.

SCEA utilizes DCEA with Hausdorff distance operator converting DARS to unevenly time series $\{x\}_{n}$. The time series obtained is also a similarity series. Then SCEA computes the convergence degree series of $\{x\}_{n}$, which is $Q$ in Algorithm II, from two perspectives: the standard-deviation and speed-changing of $x_{i}$ in window $w$. Compare to DCEA, SCEA firstly utilizes sliding method to alleviate the noise factor in DARS; and secondly SCEA depicts the convergence trend in DARS from two ways: value-changing and speedchanging, enhancing the accuracy and objectiveness of the process of disaster decision.

\section{Experiments}

In this section, we arrange two experiments to demonstrate the effectiveness of proposed algorithms. Experiments use 4 earthquake disasters in China during 2013 2015, as shown in TABLE 1. Experiment I and II testify the effectiveness of DCEA and SCEA.

Table 1: Disaster events

\begin{tabular}{|c|c|c|c|c|c|}
\hline NO. & LOC & Mag. & Time & Assess count & Assess duration \\
\hline A & LuShan,SiChuan & 7.0 & $2013-04-2008: 02$ & 277 & $7.2 \mathrm{~h}$ \\
\hline B & MinXian,GanSu & 6.6 & $2013-07-2207: 45$ & 214 & $6.9 \mathrm{~h}$ \\
\hline C & KangDing,SiChuan & 6.3 & $2014-11-2216: 55$ & 196 & $6.7 \mathrm{~h}$ \\
\hline D & ALaShan,NeiMeng & 5.8 & $2015-04-1515: 39$ & 97 & $4.5 \mathrm{~h}$ \\
\hline
\end{tabular}

Based on above disaster events, experiment I and II demonstrate the effectiveness of DCEA and SCEA, respectively. Experiment I demonstrate the effectiveness of DCEA and Experiment II demonstrates the effectiveness of SCEA and the superiority compared to the DCEA. 


\subsection{Experiment I}

In this experiment, we use Hausdorff distance as the distance operator of DCEA. We set convergence threshold $\xi_{D}$ of DARS to 0.5 . In the given condition, the convergence timestamp of DCEA is $6.5 \mathrm{~h}, 6.6 \mathrm{~h}, 4.5 \mathrm{~h}$ and $3 \mathrm{~h}$. The results of Experiment I is shown in Fig. I.
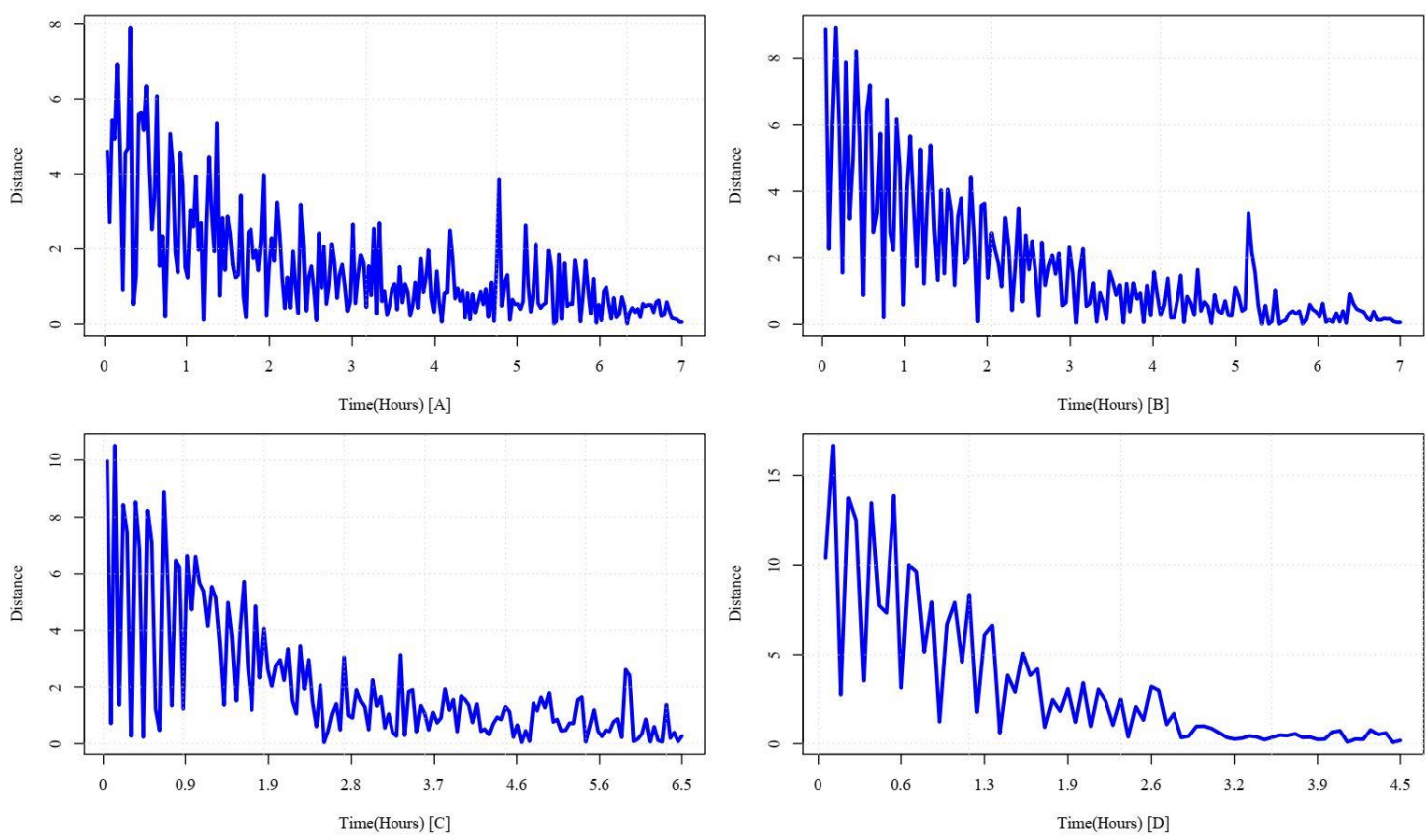

Fig. 1: Results of DCEA

Fig. A in Fig. 1 is the DCEA series of disaster A. It can be seen that the iterative assessment lasted about 7 hours in total. The distance changing between the seismic intensity impact field assessment results converged to zero over time, indicating that the DCEA algorithm is effective. The similar trends can be seen in other figures as well.

\subsection{Experiment II}

This experiment is designed to testify the effectiveness and superiority of SCEA. The start time is set to the disaster occurred. Time window $w=10 \mathrm{~min}$, step $s=5 \mathrm{~min}$, time lag $k=10 \mathrm{~min}$ and convergence threshold $\xi_{D}=0.5$. The results of the SCEA for the four disasters are shown in Fig. 2.
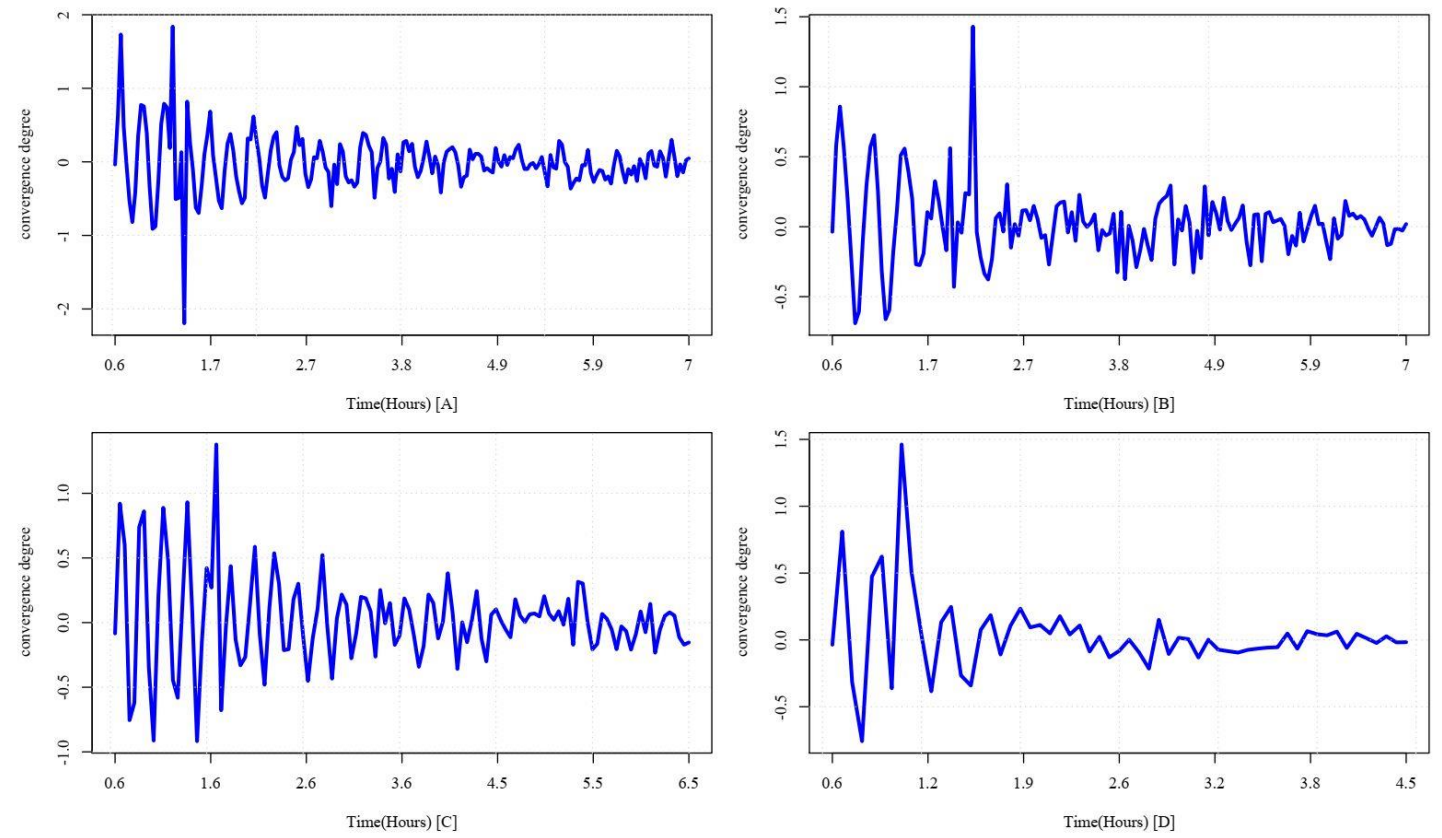

Fig. 2: Results of SCEA 
In the results of DCEA, although disaster events $\mathrm{A}$ and $\mathrm{B}$ show obvious convergence trends about 5 fours after disaster occurred, there were anomalous changes of varying degrees in the same period, which were considered to be caused by the "dirty data" in the data sources. The SCEA algorithm solves the problem more well, disaster A and B do not show any abnormal fluctuations at the relevant moment points, and the convergence trend is obvious.

Decision timestamp (i.e. convergence timestamp) obtained from SCEA for 4 disaster events are: 4.0h, $4.9 \mathrm{~h}, 3.5 \mathrm{~h}$ and $2.6 \mathrm{~h}$. It is consistent with the practical emergency decision moment of related disaster given in [6] and [7]. The experiment results are consistent with the experience of experts, managers and on-site rescuers. The SCEA is able to solve the problem of optimizing emergency decision time constraints. Relevant experimental results demonstrate the effectiveness of the SCEA algorithm.

\section{Conclusion}

To cope with the problem of optimizing decision time constraints, we proposed a range of models and methods based on the speed changing of disaster assessment result series (DARS). We firstly propose a theoretical model, which is the convergence trend in DARS, through the limit method in mathematics, and give a range of definitions of disaster objects. Secondly, a distance-based convergence extraction algorithm DCEA is proposed to convert DARS into unevenly time series using Hausdorff as the distance operator. The advantages and limitations of DCEA are analyzed and summarized. Based on above work, we continually propose an optimized algorithm SCEA to solve the time optimizing problem. Experiments on real disaster events demonstrates the effectiveness and superiority of proposed methods.

\section{Acknowledgment}

Authors thank the anonymous reviewers for their insightful and constructive comments. This research work is supported by grants from the National Natural Science Foundation of China (grant number 41631073).

\section{References}

[1] Panakkat A, Adeli H. Recurrent Neural Network for Approximate Earthquake Time and Location Prediction Using Multiple Seismicity Indicators. Computer-aided Civil and Infrastructure Engineering, 2009, 24(4): $280-292$.

[2] Xing Zhengzheng, Pei Jian, Yu P S, et al. Early prediction on time series: a nearest neighbor approach. International joint conference on artificial intelligence, 2009: 23(1):1297-1302.

[3] Dzwinel W, Yuen D A, Boryczko K, et al. Cluster Analysis, Data mining, Multi-dimensional Visualization of Earthquakes over Space, Time and Feature Space. Nonlinear Processes in Geophysics, 2008, 12(1):117-128.

[4] Azam F, Sharif M, Yasmin M, et al. Artificial intelligence-based techniques for earthquake prediction: a review. Sci Int, 2014, 26(4): 1495-1502.

[5] Su Y, Chelluboina S, Hahsler M, et al. A New Data Mining Model for Hurricane Intensity Prediction. IEEE International Conference on Data Mining Workshops. IEEE, 2011:98-105.

[6] Wang H.Y. Timing characteristics of key emergency response operations during the earthquake response period. North China Earthquake Sciences, 2014, 32(1), 59-64

[7] Wang. H.Y. Study of critical time phases during earthquake response. Journal of Catastrophology, 2013,28(03):166-169+197 